\title{
Differences in Axial Length and IOL Power Based on Alternative A-Scan or Fellow-Eye Biometry in Macula-Off Rhegmatogenous Retinal Detachment Eyes
}

Rui Liu · Hongrong Li • Qingchen Li (D)

Received: August 22, 2021 / Accepted: November 23, 2021 / Published online: December 8, 2021

(C) The Author(s) 2021

\section{ABSTRACT}

Introduction: This study was performed to observe the potential refractive prediction error based on alternative A-scan ultrasound and fellow-eye biometry for phacovitrectomy in macula-off rhegmatogenous retinal detachment (RRD) eyes.

Methods: Phakic macula-off RRD eyes without axial length (AL) measurements obtained using IOLMaster were included. Vitrectomy without lens extraction was performed for RRD repair.

R. Liu

Department of Ophthalmology, Shanghai Jing'an District Shibei Hospital, Shanghai 200443, China

H. Li

Aier Eye Hospital Group, Hangzhou Aier Eye Hospital, Hangzhou 311202, Zhejiang Province, China

Q. Li $(\bowtie)$

Department of Ophthalmology and Vision Science, Eye, Ear, Nose and Throat Hospital of Fudan University, 83\# Fenyang Road, Xuhui District, Shanghai 200031, China

e-mail: qcli16@fudan.edu.cn

\section{Q. Li}

Key Laboratory of Myopia of State Health Ministry, Key Laboratory of Visual Impairment and Restoration of Shanghai, Shanghai 200031, China

Q. Li

Laboratory of Myopia, Chinese Academy of Medical Sciences, Shanghai 200031, China
Preoperative AL was measured using alternative A-scan ultrasound (AL-US). Postoperative AL was obtained in eyes with silicone oil tamponade (AL-SO) and preoperative fellow-eye biometry (AL-FE) using IOLMaster. Other eyes that faced the same preoperative situation but underwent phacovitrectomy based on felloweye biometry were recruited as controls.

Results: AL-US, AL-FE, and AL-SO were $25.39 \pm 2.14 \mathrm{~mm}, \quad 25.85 \pm 2.16 \mathrm{~mm} \quad$ and $26.08 \pm 2.53 \mathrm{~mm}$, respectively. The Bland-Altman agreement among AL-US, AL-FE and AL-SO was good $(95.5 \%, 21 / 22$ of cases were in the LoA). The mean IOL power calculated using ALUS (Power-US), AL-FE (Power-FE) and AL-SO (Power-SO) was $16.81 \pm 7.19 \mathrm{D}, 14.74 \pm 6.95 \mathrm{D}$ and $13.54 \pm 8.32 \mathrm{D}$, respectively. The difference between AL-US and AL-SO was significant $(P<0.05)$, while that between AL-FE and AL-SO was not $(P>0.05)$. The difference between Power-US and Power-SO was significant $(P<0.05)$, while that between Power-FE and Power-SO was not $(P>0.05)$. Nine eyes underwent phacovitrectomy based on fellow-eye biometry and had a final postoperative myopic shift of $0.64 \pm 0.78 \mathrm{D}$.

Conclusions: Alternative A-scan ultrasound led to a significant difference in $\mathrm{AL}$ and a prediction error in IOL power, while fellow-eye biometry provided similar results to silicone oil-filled eyes after RRD repair. 
Keywords: Retinal detachment; Ocular biometry; Refractive error; Fellow eye; Axial length

\section{Key Summary Points}

The potential refractive prediction error based on alternative A-scan ultrasound and fellow-eye biometry for phacovitrectomy was analysed in maculaoff RRD eyes without the preoperative IOLMaster-measured axial length (AL).

The alternative preoperative AL measured with A-scan ultrasound would be significantly underestimated in this special situation, while the AL of the fellow eye is more similar to the AL in eyes filled with silicone oil tamponade after RRD repair.

The calculated IOL power based on A-scan ultrasound was significantly greater than that based on data measured in silicone oil-filled eyes.

A real-world series of 9 eyes that faced the same preoperative situation but underwent phacovitrectomy based on fellow-eye biometry showed a postoperative myopic shift of $0.64 \pm 0.78$ D.

\section{INTRODUCTION}

Rhegmatogenous retinal detachment (RRD) is a common retinal disease with an incidence of one in 10,000 people per year that often causes visual field defects and moderate to severe visual impairment [1]. Surgery is the only therapeutic approach, and surgical techniques and instrumentation developed in recent decades have led to a high primary reattachment rate [2]. Indeed, pars plana vitrectomy (PPV) is one of the most effective procedures for the treatment of RRD, with a mean postoperative anatomical success rate of over 90\% [3].
However, PPV has a higher incidence of cataract progression than scleral buckling. A variety of mechanisms might contribute to cataract progression: surgery can induce an inflammatory response, as demonstrated by an increase in aqueous flare values; or, in the absence of vitreous gel, molecular oxygen from the retinal vasculature can reach the lens and promote oxidative damage to the lens nucleus, an increase in light scattering, and nuclear sclerotic cataracts [4]. Due to postoperative complications involving the crystalline lens, more than half of phakic patients who underwent vitrectomy for RRD repair subsequently needed cataract surgery within 1 year [5]. Thus, combined phacovitrectomy was encouraged for patients aged 50 or older due to the lower cost of surgery, reduced complications and better visual outcome [6-9]. In $10-17 \%$ of cases of RRD with macular involvement, it was impossible to measure the axial length (AL) using optic biometry (IOLMaster) because of the limitations of the machine, such as dense media opacity, poor fixation by the patients or the lack of an available machine [10-12]. Rahman et al. suggested that $\mathrm{AL}$ measured by acoustic biometry (A-scan ultrasound, US) could provide an alternative intraocular lens (IOL) power calculation [12], and El-Khayat et al. reported that felloweye biometry is also recommended [13], although, to our knowledge, there has been no published report on outcomes. On the other hand, studies (including our previous work) have shown that both underestimation of the $\mathrm{AL}$ and anterior displacement of the IOL potentially contribute to a postoperative myopic shift after phacovitrectomy [14, 15]. Most studies that have focused on myopic shifts after phacovitrectomy have excluded cases in which the preoperative IOLMaster measurement was absent. Therefore, we conducted the present study to focus on this commonly ignored but not rare situation.

Here, we assessed the agreement in $\mathrm{AL}$ and predicted IOL power of macula-off RRD eyes among preoperative US, postoperative IOLMaster and fellow eyes in order to evaluate the potential refractive errors after phacovitrectomy based on alternative US or contralateral 
measurements when preoperative IOLMaster data were absent.

\section{METHODS}

\section{Design and Participants}

This retrospective, self-controlled study was conducted in Shanghai Jing'an District Shibei Hospital between September 2018 and December 2020. All study procedures were performed in accordance with the tenets of the Declaration of Helsinki and its amendments. The ethics committee of Shanghai Jing'an District Shibei Hospital approved this study. Written informed consent was obtained from all the participants for publication of their clinical data.

Phakic macula-off RRD eyes in which ocular biometric measurements could not be obtained using IOLMaster were included; the eyes underwent 23-gauge PPV with silicone oil (SO) tamponade but not lens extraction. Eyes with a history of anisometropia and eyes with scleral buckling surgery, recurrent retinal detachment or other ocular problems that may have affected biometric measurements, including corneal scarring and lens dislocation, were excluded [16].

Other patients who faced the same preoperative situation but underwent phacovitrectomy based on fellow-eye biometry for RRD repair were recruited as controls at least 3 months after SO removal. During follow-up, after excluding those with recurrent RRD or other postoperative complications, comprehensive refractive measurements were performed to obtain the spherical dioptre. The mean spherical error was defined as the difference in spherical dioptre between the postoperative and expected refractive outcomes.

\section{Surgical Procedure}

A standard 23-gauge PPV using the CONSTELLATION $^{\circledR}$ Vision 106 System (Alcon Laboratories, Inc.) was performed in each case. During the PPV, the RESIGHT ${ }^{\mathrm{TM}}$ Fundus Viewing System (Carl Zeiss Meditec Inc.) was used.
Core vitrectomy, midperipheral vitrectomy and vitreous base shaving under scleral depression were performed to remove the vitreous. Perfluorocarbon liquid (Perfluoron; Alcon Laboratories, Inc.) was used in some cases, depending on the extent of retinal detachment. Endolaser photocoagulation was performed around areas with retinal breaks, and fluid-air exchange was performed before silicone oil injection (Oxane 5700 centistokes; Bausch \& Lomb Inc., Waterford, Ireland).

\section{Measurement Techniques}

Preoperative AL values of RRD eyes were measured using A-scan ultrasound biometry (UD6000 Ultrasonic A/B scanner biometer; Tomey Corporation, Nagoya, Japan), as previously described [17], instead of using IOLMaster. Before SO removal, an optical coherence tomography scan was performed to ensure that the macula was attached in both eyes, and then the $\mathrm{AL}$ values of postoperative RRD eyes and fellow eyes were obtained with IOLMaster (Model 700, Carl Zeiss Meditec Ltd, Jena, Germany) using phakic and SO-filled phakic eye programs, respectively. All the AL measurements performed in this study were repeated three times by experienced examiners. The IOL power was calculated based on the constant A of the AcrySof ${ }^{\circledR}$ IQ ReSTOR ${ }^{\circledR}$ SN6AD1 IOL (Alcon Laboratories, Inc., Fort Worth, USA) with the SRK/T formula.

\section{Statistical Analysis}

Continuous variables are expressed as the mean \pm standard deviation. Statistical analyses were performed using the software package SPSS Statistics 26.0 for Windows (SPSS Inc., Chicago, IL, USA). Bland-Altman plots were drawn using MedCalc 15.2.2 (MedCalc Software bvba, Ostend, Belgium). Statistical significance was set at $P<0.05$. 


\section{RESULTS}

Data from 22 eyes of 22 patients (12 males and 10 females) with an age range from 36 to 65 years (mean: $51.6 \pm 8.3$ years) were enrolled. The mean duration of SO tamponade was $4.3 \pm 0.8$ months. The mean $\mathrm{AL}$ in eyes with RRD measured by A-scan ultrasound (AL-US) was $25.39 \pm 2.14 \mathrm{~mm}(22.59-31.53 \mathrm{~mm})$, the mean AL of fellow eyes (AL-FE) was $25.85 \pm 2.16 \mathrm{~mm}(22.47-31.56 \mathrm{~mm})$, and the mean AL in eyes with SO tamponade after RRD repair (AL-SO) was $26.08 \pm 2.53 \mathrm{~mm}$ (22.75-32.81 mm). Independent-samples $t$ tests revealed that there was no significant difference in AL-US, AL-FE or AL-SO between the sexes (all $P>0.05)$.

The mean IOL power calculated using AL-US (Power-US) was $16.81 \pm 7.19$ dioptres (D), the mean IOL power calculated using AL-FE (Power$\mathrm{FE}$ ) was $14.74 \pm 6.95 \mathrm{D}$, and the mean IOL power calculated using AL-SO (Power-SO) was $13.54 \pm 8.32$ D. Bland-Altman plots demonstrated that the agreement between AL-US and AL-SO was good since $95.5 \%(21 / 22)$ of samples were included in the $95 \%$ limits of agreement (LoA), and the agreement between AL-FE and AL-SO $(95.5 \%, 21 / 22$ in LoA) was similar, as shown in Fig. $1 \mathrm{~A}$ and $\mathrm{B}$.

However, repeated-measures ANOVA showed that the difference between AL-US and AL-SO was statistically significant $(P<0.05)$, while that between AL-FE and AL-SO was not $(P>0.05$, Fig. 1C). Correspondingly, the difference between Power-US and Power-SO was statistically significant $(P<0.05)$, and the mean difference (Power-US - Power-SO) was $3.27 \pm 3.79 \mathrm{D}$, while that between Power-FE and Power-SO was not significant $(P>0.05$, Fig. 1D).

Nine eyes of 9 patients $(4$ males and 5 females) with a mean age of $58.1 \pm 6.5$ years were recruited as controls. They were also phakic macula-off RRD eyes without preoperative IOLMaster measurements. Standard 23-gauge phacovitrectomy with SO tamponade was performed for each of them, and the implanted IOLs were all AcrySof ${ }^{\circledR}$ IQ ReSTOR ${ }^{\circledR}$ SN6AD1 IOLs, for which the power was calculated with the SRK/T formula based on fellow-eye biometry. The mean follow-up period after SO removal was $8.9 \pm 3.1$ months. The mean spherical error of those 9 eyes showed a postoperative myopic shift of $0.64 \pm 0.78 \mathrm{D}$.

\section{DISCUSSION}

The AL of an eye, which is measured as the distance from the cornea to the inner limiting membrane using A-scan US or the distance from the cornea to retinal pigment epithelium using IOLMaster [18], is the most crucial parameter in the IOL power calculation. A 1-mm change in the AL corresponds to an approximately 2.7-D refractive error in the IOL power [19]. Nepp et al. reported that the mean difference between the US-measured AL and the IOLMaster-measured AL in SO-filled eyes was $0.4 \mathrm{~mm}$, and only $46 \%$ of cases had a value of less than $0.3 \mathrm{~mm}$, while the value was greater than $1 \mathrm{~mm}$ in $26 \%$ [20]. Thus, we measured the AL values of these SO-filled eyes and normal fellow eyes in the present study by means of IOLMaster to achieve more accuracy and less deviation [16]. Our results showed that the $\mathrm{AL}$ values of SO-filled eyes after RRD repair were comparable with those of fellow eyes, so it was not surprising that the IOL power calculated using AL-SO was similarly comparable to that calculated using AL-FE. However, there were significant differences in both AL and IOL power between USmeasured preoperative data for macula-off RRD eyes and IOLMaster-measured postoperative data for SO-filled eyes. The mean AL-US was approximately $0.68 \mathrm{~mm}$ shorter than the mean AL-SO, and the mean Power-US was accordingly 3.27 D greater than the mean Power-SO. Thus, there would be a clinically significant myopic shift after SO removal if we performed phacovitrectomy for RRD repair based on the alternative A-scan-based IOL power calculation.

Phacovitrectomy is a safe and effective procedure to treat RRD. Kang et al. and Sakamoto et al. confirmed that there is a tolerable biometric error after the treatment of RRD with phacovitrectomy in macula-sparing cases, with comparable results obtained with acoustic and optic biometry [21, 22]. Although the 

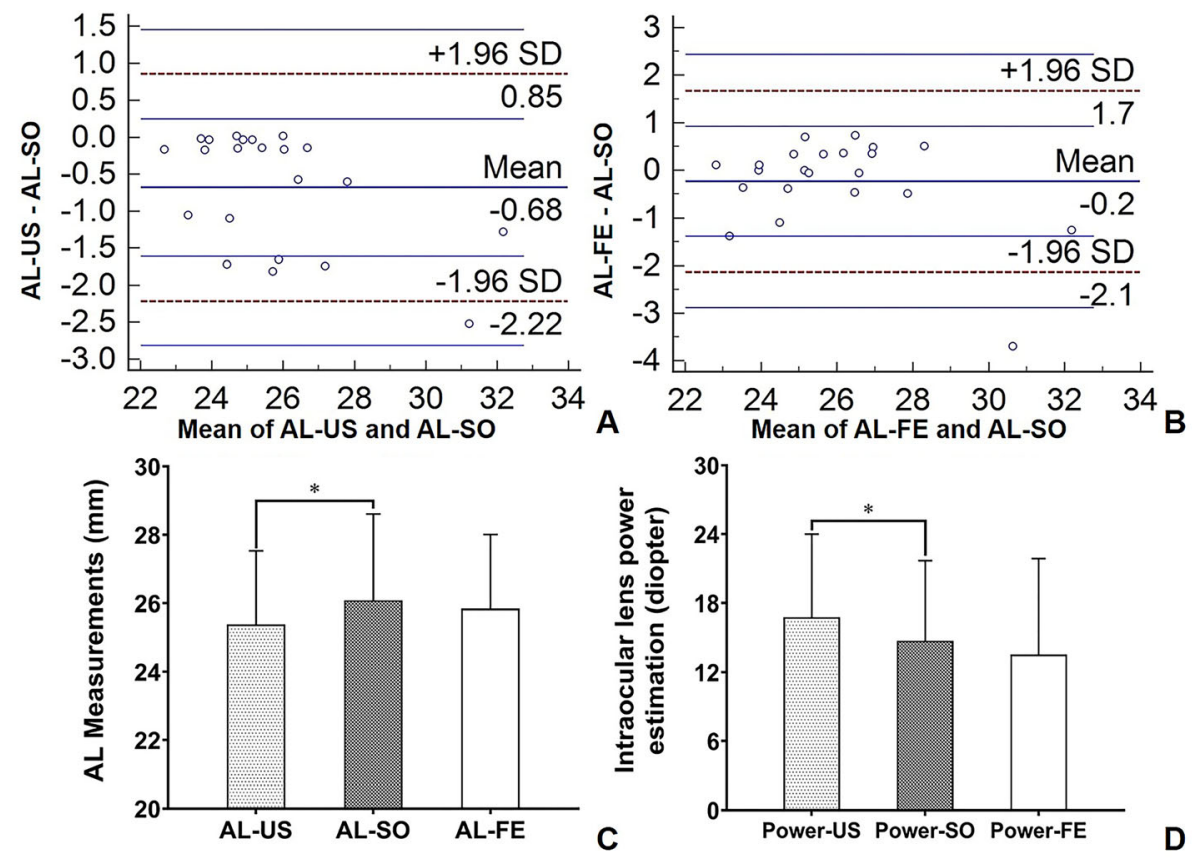

Fig. 1 Bland-Altman plots for AL-US and AL-SO (A) and for AL-FE and AL-SO (B). The bold solid line indicates the mean difference; the dotted lines represent the 95\% limits of agreement (LoA), and the thin solid lines represent the $95 \%$ CI of the upper/lower limits of the LoA. Differences in axial length (C) and IOL power estimation (D) among RRD eyes, SO-filled eyes and fellow eyes (repeated-measures ANOVA, $\left.{ }^{*} P<0.05\right)$ ). AL-US

anatomical and functional results were comparable with those obtained with single PPV and delayed cataract surgery, the refractive outcomes were less favourable and shifted towards myopia, especially in macula-off cases [23]. When the macula was involved, there was a mean prediction error of $-1.22 \pm 2.32 \mathrm{D}$ for $\mathrm{RRD}$ eyes and a mean prediction error of $0.01 \pm 1.09 \mathrm{D}$ for fellow eyes [13]. Pongsachareonnont et al. attributed this difference to an underestimation of $0.59 \pm 0.90 \mathrm{~mm}$ in $\mathrm{AL}$ measurements performed by IOLMaster [24]. Kim et al. found that the underestimation was associated with the macular retinal detachment height [11]. Unlike the above studies, the present study focused on a special situation. Rahman et al. found that IOLMaster could provide $\mathrm{AL}$ measurements in less than one-quarter $(13 / 54,24.1 \%)$ of the cases [10]. axial length of RRD eye measured by A-scan, $A L-S O$ axial length of eye with silicone oil tamponade for RRD repair, $A L-F E$ axial length of fellow eye, Power-US estimated IOL power of RRD eye measured by A-scan, Power-SO estimated IOL power of eye with silicone oil tamponade for RRD repair, Power-FE estimated IOL power of fellow eye

Patients with worse vision, greater central macular thickness and shallow anterior chambers required more caution since their eyes were prone to inaccurate preoperative biometry [25]. Since there were no preoperative IOLMaster data to use as a reference, we could not calculate a user-adjusted AL by combining acoustic and optic biometry as previously described [12]. Therefore, delayed cataract surgery or phacovitrectomy using contralateral AL might be recommended. In the present study, we also retrospectively reviewed 9 patients who faced the same preoperative situation but underwent phacovitrectomy based on fellow-eye biometry in a real-world setting, and the mean spherical error between their postoperative and expected refractive outcomes was $-0.64 \pm 0.78 \mathrm{D}$. This postoperative myopic shift was expected and comparable with eyes reported in previous 
studies that underwent phacovitrectomy for RRD repair based on preoperative IOLMaster data.

The results of this study should be interpreted with its limitations in mind. All the participants we recruited underwent SO tamponade, but interference with biometry (such as underfilling/overfilling or emulsification of the SO) was not entirely quantified. Also, some characteristics of retinal detachment that might be associated with the accuracy of AL measurement, such as macular detachment height, were not controlled.

\section{CONCLUSION}

Based on the present study, if IOLMaster cannot be performed in eyes with macula-off RRD, the use of ultrasound might lead to a refractive prediction error in IOL power. Phacovitrectomy using fellow-eye biometry as well as secondary IOL implantation could be the better choice in this situation.

\section{ACKNOWLEDGEMENTS}

We thank the participants of the study.

Funding. The Rapid Service Fee of this study was supported by Advanced and Appropriate Technology Promotion Project of Shanghai Health Commission (2019SY012), and Shanghai Medical Key Special Construction Project (ZK2019B27).

Authorship. All named authors meet the International Committee of Medical Journal Editors (ICMJE) criteria for authorship for this article, take responsibility for the integrity of the work, and give their approval for this version to be published.

Authorship Contributions. Rui Liu collected the data and wrote the initial draft of the manuscript. Hongrong Li contributed to interpretation of data and assisted in the preparation and reviewing of the manuscript. Qingchen $\mathrm{Li}$ conceived the study and revised the final manuscript. All authors approved the final version of the manuscript and agree to be accountable for all aspects of the work in ensuring that questions related to the accuracy or integrity of any part of the work are appropriately investigated and resolved.

Compliance with Ethics Guidelines. All procedures performed were in accordance with the ethical standards of the Ethics Committee of the Declaration of Helsinki and its later amendments or comparable ethical standards. The Ethics Committee of Shanghai Jing'an District Shibei Hospital approved this study. Written informed consent was obtained from all the participants for publication of their clinical data.

Disclosures. The authors Rui Liu, Hongrong $\mathrm{Li}$, and Qingchen $\mathrm{Li}$, confirm that they have nothing to disclose.

Data Availability. The datasets used and/or analyzed during the current study available from the corresponding author on reasonable request.

Open Access. This article is licensed under a Creative Commons Attribution-NonCommercial 4.0 International License, which permits any non-commercial use, sharing, adaptation, distribution and reproduction in any medium or format, as long as you give appropriate credit to the original author(s) and the source, provide a link to the Creative Commons licence, and indicate if changes were made. The images or other third party material in this article are included in the article's Creative Commons licence, unless indicated otherwise in a credit line to the material. If material is not included in the article's Creative Commons licence and your intended use is not permitted by statutory regulation or exceeds the permitted use, you will need to obtain permission directly from the copyright holder. To view a copy of this licence, visit http://creativecommons.org/licenses/by$\mathrm{nc} / 4.0 /$. 


\section{REFERENCES}

1. Bonfiglio V, Reibaldi M, Macchi I, Fallico M, Pizzo C, Patane C, Russo A, Longo A, Pizzo A, Cillino G, et al. Preoperative, intraoperative and postoperative corticosteroid use as an adjunctive treatment for rhegmatogenous retinal detachment. J Clin Med. 2020;9(5):1556.

2. Reeves MGR, Afshar AR, Pershing S. Need for retinal detachment reoperation based on primary repair method among commercially-insured patients, 2003-2016. Am J Ophthalmol. 2021. https://doi. org/10.1016/j.ajo.2021.04.007.

3. Sultan ZN, Agorogiannis EI, Iannetta D, Steel D, Sandinha T. Rhegmatogenous retinal detachment: a review of current practice in diagnosis and management. BMJ Open Ophthalmol. 2020;5(1): $\mathrm{e} 000474$.

4. Gagliano C, Toro MD, Avitabile T, Stella S, Uva MG. Intravitreal steroids for the prevention of PVR after surgery for retinal detachment. Curr Pharm Des. 2015;21(32):4698-702.

5. Benson MD, Sia D, Seamone ME, Greve M, Hinz B, Tennant MTS, Baker C, Somani R, Ehmann DS. Phacovitrectomy for primary rhegmatogenous retinal detachment repair: a retrospective review. Retina. 2020;41(4):753-60.

6. Savastano A, Lenzetti C, Finocchio L, Bacherini D, Giansanti F, Tartaro R, Piccirillo V, Savastano MC, Virgili G, Rizzo S. Combining cataract surgery with 25-gauge high-speed pars plana vitrectomy: a prospective study. Eur J Ophthalmol. 2020;121(1): 299-304.

7. Port AD, Nolan JG, Siegel NH, Chen X, Ness SD, Subramanian ML. Combined phaco-vitrectomy provides lower costs and greater area under the curve vision gains than sequential vitrectomy and phacoemulsification. Graefe's Arch Clin Exp Ophthalmol. 2020;259(1):45-52.

8. Antaki F, Milad D, Javidi S, Dirani A. Vitreoretinal surgery in the post-lockdown era: making the case for combined phacovitrectomy. Clin Ophthalmol. 2020;14:2307-9.

9. Auchere Lavayssiere C, Lux AL, Beraud G, Degoumois A, Billotte C, Denion E. Bag-in-the-lens implantation helps avoid posterior synechiae of the iris after phacovitrectomy. J Cataract Refract Surg. 2019;45(10):1386-92.

10. Rahman R, Bong CX, Stephenson J. Accuracy of intraocular lens power estimation in eyes having phacovitrectomy for rhegmatogenous retinal detachment. Retina. 2014;34(7):1415-20.
11. Kim YK, Woo SJ, Hyon JY, Ahn J, Park KH. Refractive outcomes of combined phacovitrectomy and delayed cataract surgery in retinal detachment. Can J Ophthalmol. 2015;50(5):360-6.

12. Rahman R, Kolb S, Bong CX, Stephenson J. Accuracy of user-adjusted axial length measurements with optical biometry in eyes having combined phacovitrectomy for macular-off rhegmatogenous retinal detachment. J Cataract Refract Surg. 2016;42(7):1009-14.

13. El-Khayat AR, Brent AJ, Peart SAM, Chaudhuri PR. Accuracy of intraocular lens calculations based on fellow-eye biometry for phacovitrectomy for macula-off rhegmatogenous retinal detachments. Eye (Lond). 2019;33(11):1756-61.

14. van der Geest LJ, Siemerink MJ, Mura M, Mourits MP, Lapid-Gortzak R. Refractive outcomes after phacovitrectomy surgery. J Cataract Refract Surg. 2016;42(6):840-5.

15. Liu R, Li Q. Changes in ocular biometric measurements after vitrectomy with silicone oil tamponade for rhegmatogenous retinal detachment repair. BMC Ophthalmol. 2020;20(1):360.

16. Kunavisarut $\mathrm{P}$, Poopattanakul $\mathrm{P}$, Intarated $\mathrm{C}$, Pathanapitoon K. Accuracy and reliability of IOL master and A-scan immersion biometry in silicone oil-filled eyes. Eye (Lond). 2012;26(10):1344-8.

17. Alvani A, Pakravan M, Esfandiari H, Yaseri M, Yazdani S, Ghahari E. Biometric changes after trabeculectomy with contact and non-contact biometry. Optom Vis Sci. 2016;93(2):136-40.

18. Kim M, Kim HE, Lee DH, Koh HJ, Lee SC, Kim SS. Intraocular lens power estimation in combined phacoemulsification and pars plana vitrectomy in eyes with epiretinal membranes: a case-control study. Yonsei Med J. 2015;56(3):805-11.

19. Olsen T. Calculation of intraocular lens power: a review. Acta Ophthalmol Scand. 2007;85(5): 472-85.

20. Nepp J, Krepler K, Jandrasits K, Hauff W, Hanselmayer G, Velikay-Parel M, Ossoinig KC, Wedrich A. Biometry and refractive outcome of eyes filled with silicone oil by standardized echography and partial coherence interferometry. Graefe's Arch Clin Exp Ophthalmol. 2005;243(10):967-72.

21. Kang TS, Park HJ, Jo YJ, Kim JY. Long-term reproducibility of axial length after combined phacovitrectomy in macula-sparing rhegmatogenous retinal detachment. Sci Rep. 2018;8(1):15856.

22. Sakamoto M, Yoshida I, Sodeno T, Sakai A, Masahara $\mathrm{H}$, Maeno $\mathrm{T}$. Postoperative refractive 
prediction error measured by optical and acoustic biometry after phacovitrectomy for rhegmatogenous retinal detachment without macular involvement. J Ophthalmol. 2019. https://doi.org/10. $1155 / 2019 / 5964127$.

23. Tan A, Bertrand-Boiche M, Angioi-Duprez K, Berrod JP, Conart JB. Outcomes of combined phacoemulsification and pars plana vitrectomy for rhegmatogenous retinal detachment: a comparative study. Retina. 2020;41(1):68-74.

24. Pongsachareonnont P, Tangjanyatam S. Accuracy of axial length measurements obtained by optical biometry and acoustic biometry in rhegmatogenous retinal detachment: a prospective study. Clin Ophthalmol. 2018;12:973-80.

25. Tranos PG, Allan B, Balidis M, Vakalis A, Asteriades S, Anogeianakis G, Triantafilla M, Kozeis N, Stavrakas $\mathrm{P}$. Comparison of postoperative refractive outcome in eyes undergoing combined phacovitrectomy vs cataract surgery following vitrectomy. Graefes Arch Clin Exp Ophthalmol. 2020;258(5): 987-93. 